A detector for the induced pseudoscalar coupling constant

Ericson, Torleif Eric Oskar; Sens, J C

Published in Nuovo Cimento 34 (1964) 51-66

This article has been scanned with the kind permission of the Società Italiana di Fisica.

From: Angela Oleandri <oleandriessif it>

Date: October 12, 2007 4:07:47 PM GMT+02:00

Subject: Re: Request for permigsion to reproduce published material.

Dear Dr Mele,

further to your request, sent in July to Carmen Vasini (herewith attached)

I'm pleased to inform you that the Italiam Physical society is happy to
grant you permission of reproduction of the listed material for your
archival purposes at no charges.

athers at 


\title{
A Detector for the Induced Pseudoscalar Coupling Constant (*).
}

T. ERICSON and J. C. SENS

$C E R N$ - Geneva

H. P. C. RrOoD

Institute of Theoretical Physics - Groningen

(ricevuto il 15 Ottobre 1963)

\begin{abstract}
Summary. - The detection of partial muon capture rates in ${ }^{16} \mathrm{O}$ forms a sensitive detector for the induced pseudoscalar coupling constant. We here investigate the effects of nucleon velocity terms in the weak Hamiltonian and of realistic nuclear wave-functions. Their inclusion is essential for a correct treatment of the problem. In addition to the previously suggested detector level, we find that capture into the ground state of ${ }^{16} \mathrm{~N}$ permits an almost complete elimination of nuclear physics by comparison to the $\beta$-decay rate.
\end{abstract}

\section{1. - Introduction.}

Two of the following three weak processes, neutron decay, muon decay and muon capture involve nucleons and are thus directly affected by strong interactions. To arrive at a "universal » theory for the weak interactions these "strong" effects must be isolated and subtracted out. Important advances have been made in this direction by the methods of dispersion relations (DR) and by the proposal of a conserved vector current theory (CVC).

$\mathrm{DR}$ in particular predict that:

i) if the muon-bare nucleon and the electron-bare nucleon coupling constants are the same, i.e., if universality holds, then the muon-dressed nucleon

(*) This article supersedes an earlier preprint in which'several errors have been corrected. 
and electron-dressed nucleon axial vector coupling constants are nearly the same also: $g_{A}^{\mu} \approx g_{A}^{\beta}$;

ii) the one-pion intermediate state introduces a large extra pseudoscalar interaction in muon capture: $g_{p} \simeq 8 g_{A}^{\mu}$.

CVYG theory predicts: .

a) that if universality holds, then $g_{v}^{\mu} \approx g_{v}^{\beta}$;

b) that the vector interaction must be completed by a direct

$$
\pi^{+}+\left\{\begin{array}{l}
e^{-} \\
\mu^{-}
\end{array} \rightarrow \pi^{0}+v\right.
$$

interaction in order to make the nucleon current obey a conservation law similar to that in electrodynamics. This gives rise to well-known "weak magnetic " terms in the Hamiltonian.

Evidence for the induced pseudoscalar coupling is in principle present in a number of experimentally observable reactions. : These are the total muon capture rates, rates of capture into a particular final state, the angular distribution of the recoiling nucleus, the angular distribution and the polarization of neutrons from muon capture and the radiative muon capture (the spectrum, the circular polarization and the angular distribution of the photons). Insufficient knowledge of the nuclear radius, the muon and the nucleon wave-functions and the mean neutrino energy make a straightforward interpretation of some of these experiments difficult; moreover, in other cases the effect is proportional to the residual muon polarization in the $s$-state, which is known to be small $(\approx 15 \%)$. $\beta$-decay is not a good test for the induced pseudoscalar coupling since the effect is then reduced by $m_{\theta} / m_{\mu}$ (in the coupling constant) with respect to muon capture.

At present only meagre information is available on the pseudoscalar coupling constant. The neutron asymmetry following $\mu$-capture in various elements suggests a large value of $g_{P} / g_{A}$ with appreciable model-dependence ( $\left.{ }^{1}\right)$. The recently measured photon spectrum in the radiative muon capture ( $\left.{ }^{2}\right)$ varies significantly with $g_{p}$. The ratio of this capture rate to the total capture rate depends only weakly on nuclear structure, the main source of uncertainty being the mean neutrino momentum in total capture $\left(^{3}\right)$.

(') V: S. Evseev, V. S. Roganov, V. A. Chernogorova, M. M. SzymezaK and Chang Run-Hwa: 1962 International Conference on High-Energy Physics, CERN p. 425 .

(2) M. Conversi, R. Diebold and L. Dr LeLLA : International Conference on Fundamental Aspects of Weats Interactions (Brookhaven, 9-11 September, 1963).

( ${ }^{3}$ H. P. C. Rood and H. A. Tolmowk: Phys. Lett., 6, 121 (1963) 
It has been pointed out by SHAPIRo and BLoKHINTSEv ( $\left.{ }^{4}\right)$ that the ratio of the partial muon capture in ${ }^{16} \mathrm{O}$ to two particular excited states in ${ }^{16} \mathrm{~N}$ is strongly dependent on both the sign and magnitude of $g_{p}$ while independent on nuclear matrix elements. Their considerations have been made with strongly simplifying assumptions. In this article we will make a simple reconsideration of their calculation; in particular admixtures in nuclear wave-functions and nucleon velocity terms are taken into account. These terms are expected to be of importance due to the forbiddenness of the transition. While the main conclusion of great sensitivity to $g_{p}$ remains unaltered, the ratio is no longer model-independent.

In Sect. 2 we give the complete square of the nuclear matrix element for partial capture (including linear nucleon velocity terms in the effective Hamiltonian). In Sect. 3 a simple derivation of the Shapiro-Blokhintsev result is given. We also discuss the general conditions for maximum sensitivity to $g_{P}$. Section 4 includes the effects of nuclear velocity terms and of admixture to the pure $(j j)$-coupling scheme. Section 5 gives a discussion of results and a comparison to experiment.

\section{2. - Matrix element for partial eapture.}

The probability for capture of a negative muon from the $K$ shell of nucleus a with formation of nucleus $\mathrm{b}$ is to first order in $p / M$ given by

$$
\Lambda_{\mu \mathrm{e}}(\mathrm{a} \rightarrow \mathrm{b})=\frac{\nu^{2}}{2 \pi}\left|\varphi_{\mu}\right|_{\mathrm{av}}^{2} M_{\mathrm{ab}}^{2} .
$$

Here, $M_{\mathrm{ab}}^{2}$ can be split into three parts (we assume the initial spin to be zero)

$$
M_{\mathrm{ab}}^{2} \equiv \sum_{M_{\mathrm{b}}} \int \frac{\mathrm{d} \hat{v}}{4 \pi}\left\{A_{0}+A_{1}+A_{2}\right\}
$$

with

$$
\begin{aligned}
& A_{0}=G_{V}^{2}\left|\int 1\right|^{2}+G_{A}^{2}\left|\int \sigma\right|^{2}+\left(G_{P}^{2}-2 G_{P} G_{A}\right)\left|\int \hat{\nu} \cdot \sigma\right|^{2} \\
& A_{1}=-G_{V} g_{V}\left\{\left(\int 1\right)^{*}\left(\int \frac{\boldsymbol{p} \cdot \hat{v}}{M}\right)+\text { c.c. }\right\}-\left(G_{A}-G_{P}\right) g_{A}\left\{\left(\int \hat{v} \cdot \sigma\right)^{*}\left(\int \frac{p \cdot \sigma}{M}\right)+\text { c.c. }\right\}+ \\
&+G_{A} g_{V} i \hat{v}:\left\{\left(\int \sigma\right)^{*} \times\left(\int \frac{\boldsymbol{p}}{M}\right)-\text { c.c. }\right\}
\end{aligned}
$$

$$
A_{2}=g_{\Delta}^{2}\left|\int \frac{\sigma \cdot p}{M}\right|^{2}+g_{V}^{2}\left|\int \frac{p}{M}\right|^{2}
$$

(4) I. S. Shapiro and L. D. Blokhintsev: Soviet Physics, J.E.T.P., 12, 775 (1961). 
We have used the abbreviation

$$
\int \mathcal{O}=\left\langle M_{\mathrm{b}}\left|\sum_{i} \tau_{i}^{-} \exp \left[-i v \cdot r_{i}\right] \mathcal{O}_{i}\right| M_{\mathrm{a}}\right\rangle
$$

for the matrix element of a one-particle operator $\mathcal{O}_{i}$ between an initial state $M_{\mathrm{a}}$ and a final state $M_{\mathrm{b}}$. The three terms $A_{0}, A_{1}$ and $A_{2}$ refer to increasing powers of $p / M$. Furthermore, the following notations are used:

$\tau_{i}^{-} \quad=$ isospin operator changing the $i$-th proton state into the $i$-th neutron state,

$\nu \quad=\nu \hat{v}=3$-momentum of the neutrino for transition $a \rightarrow b$,

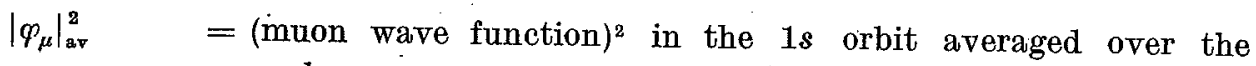
nucleus,

$G_{\Delta} \quad=g_{V}(\lambda-\mu x)$,

$G_{p} \quad=g_{V}[\lambda(\varrho-1)-\mu] x$,

$\varrho \quad=g_{p} / g_{A}$,

$\lambda \quad=g_{\Delta} / g_{V}$,

$\mu \quad=1+\mu_{\mathrm{v}}-\mu_{\mathrm{n}}$ (assuming CVC theory),

$x \quad=i / 2 M$,

$M \quad=$ nucleon mass,

$g_{v}, g_{A}$ and $g_{p}=$ the vector, axial vector and (induced) pseudoscalar coupling constants in $\mu$ capture,

$\sigma_{i} \quad=$ spin operator of the $i$-th nucleon,

$\boldsymbol{p}_{i} \quad=$ momentum operator of the $i$-th nucleon.

The squared matrix element is derived from the effective Hamiltonian as given by FuJI and PRIMakoff (5), except for the last term of $A_{1}$ in eq. (4) which was omitted in Ref. $\left(^{5}\right)$ (see, e.g., Ref. $\left(^{8}\right)$ ).

In the matrix element the mún is treated nonrelativistically. An average is made over all spin orientations of the muon, i.e., all effects of the slight residual muon polarization are ignored. Both the first-order nucleon recoil and velocity terms are taken into account.

${ }^{5}$ ) A. FuJII and H. Primakoff: Nuovo Cimento, 12, 237 (1959).

$\left(^{6}\right)$ J. R. LUTTEN, H. P. C. Rood and H. A. Tolmoer: Nucl. Phys., 41, 236 (1963);

J. DeLoRMe: Thesis, University of Lyon (1963). 


\section{3. - The Shapiro-Blokhintsev ratio.}

The spectrum of ${ }^{16} \mathrm{~N}$ is characterized by four low-lying odd-parity states of spin $0^{-}, 1^{-}, 2^{-}$, and $3^{-}$within an energy interval of $400 \mathrm{keV}$. There are no other levels below $3.53 \mathrm{MeV}$ (see Fig. 1). These states are theoretically well described ais (proton ${ }^{-1}$, neutron) states in ( $j j)$-coupling. In particular, the calculations of ELLIOTT and Fuowers predict four close-lying odd-parity levels of spin $0,1,2,3$ followed by a gap of several $\mathrm{MeV}\left({ }^{7}\right)$. The order of the four levels depends on the details of the interaction potential. The $0^{-}$and $1^{-}$states are almost puxe configurations of $\left(1 p_{\frac{1}{2}}^{-1}, 2 s_{\frac{1}{2}}\right)$, while the $2^{-}$and $3^{-}$states are almost pure $\left(1 p_{\frac{1}{2}}^{-1}, 1 d_{\frac{5}{2}}\right)$. The properties of these states are in good agreement with theory.

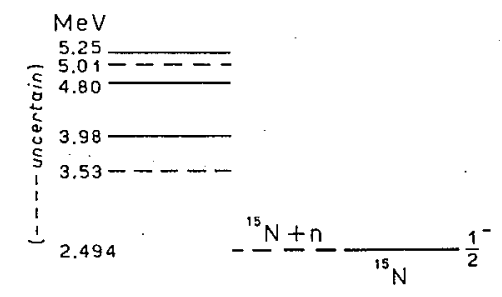

The wave-functions of the $0^{-}$ and $1^{-}$states can thus be written as a ${ }^{16} \mathrm{O}$ core with the proton-hole and neutron spins coupled to the correct total spin. They are thus $\left(^{*}\right)$

$$
|I M\rangle \equiv\left|M_{\mathrm{b}}\right\rangle=\left(\sum_{m_{1} m_{2}} C\left(\frac{1}{2} \frac{1}{2} m_{1} m_{2} \mid I M\right)\left|1 p_{\frac{1}{2}}^{-1} m_{1}\right\rangle_{\mathrm{p}}\left|2 s_{\frac{1}{2}} m_{2}\right\rangle_{\mathrm{n}}\right)
$$

where $I$ takes values 0 and 1 .

The ${ }^{16} \mathrm{O}$ ground state has spin $0^{+}$; it is magic in neutrons and protons due to the closure of the major $1 p$ shell for both. It is convenient in the calculation to write the ${ }^{16} \mathrm{O}$ ground-state wave-function as the ${ }^{16} \mathrm{O}$ core with a hole in one of its occupied states coupled to a particle in the same state so as to give zero total spin. The hole is chosen so as to match the proton hole in the ${ }^{16} \mathrm{~N}$ wave-function and hence for the $\left(\mathrm{p}_{\frac{1}{2}}\right)^{-1}$ of eq. (7), the ${ }^{16} \mathrm{O}$ wave function is

$$
|0+\rangle \equiv\left|M_{\mathrm{a}}\right\rangle=\left(\sum_{m} C\left(\frac{1}{2} \frac{1}{2} m-m \mid 00\right)\left|1 p_{\frac{1}{2}}^{-1} \dot{m}\right\rangle_{\mathrm{p}}\left|1 p_{\frac{1}{2}}-m\right\rangle_{\mathrm{p}}\right)
$$

(7) J. P. Elliote and B. H. Flowers: Proc. Roy. Soc., A 242, 57 (1957).

(*) The wave-functions (7) and (8) are in symbolic notation and are still to be antisymmetrized. 
In order to obtain the ratio of the transition rates $\left(0^{+} \rightarrow 0^{-}\right) /\left(0^{+} \rightarrow 1^{-}\right)$in the approximation made by ShapIro and BLoKHINTSEv, we put $A_{1}=A_{2}=0$ in Eq. (2), i.e., we omit all nucleon velocity terms.

On substitution of eqs. (7) and (8) into eq. (3), one notes the following:

i) the capture operators are one-particle operators and thus affect only the proton in eq. (8), not the hole, which is unchanged; therefore, $\dot{m}_{1}=m$, which links the Clebsch-Gordan coefficients,

ii) both in the $0^{-}$and ${1^{-}}^{-}$capture the change in orbital angular momentum is $\Delta l=1$, the difference being taken away by the neutrino; thus the
effective part of

$$
\exp [-i \nu \cdot r]=\sum_{l}(-i)^{l} j_{l}(v r) \sqrt{4 \pi(2 l+1)} Y_{l 0}(\hat{\nu} \cdot \hat{r}),
$$

is only the $l=1$ part, $-i j_{1}(\nu r) 2 \sqrt{3} \bar{\pi} Y_{10}(\hat{\nu} \cdot \hat{r})$,

iii) the calculation of the sum of the capture rates into the $0^{-}$and $1^{-}$, states is simplified by closure in the spins, i.e., by use of the sum rule

$$
\sum_{l M I} C\left(\frac{1}{2} \frac{1}{2} m_{1} m_{2} \mid I M\right) C\left(\frac{1}{2} \frac{1}{2} m_{1}^{\prime} m_{2}^{\prime} \mid I M\right)=\delta_{m_{2} m_{2}^{\prime}} \delta_{m_{2} m_{2}^{\prime}},
$$

which eliminates the Clebsch-Gordan coefficients.

We can evaluate $\sum_{b=0^{-} \text {and } 2^{-}}\left|M_{\mathrm{ab}}\right|^{2}$ in a straightforward way:

$$
\sum_{b=0-a n d 1^{-}}\left|M_{\mathrm{ab}}\right|^{2}=2\left|I_{1}\right|^{2} \cdot\left[G_{\bar{V}}^{2}+2 G_{A}^{2}+\left(G_{P}-G_{A}\right)^{2}\right],
$$

since $\sigma^{2}=3$ and $(\sigma \cdot \hat{v})^{2}=1 . I_{1}$ is the radial integral

$$
I_{1}=\int_{0}^{\infty} j_{1}(\nu r) \mathscr{R}_{2 s}(r) \mathscr{R}_{1 p}(r) r^{2} \mathrm{~d} r .
$$

From eq. (10) we can immediately obtain an estimate of the transition rate to the $0^{-}$and $1^{-}$states using harmonic oscillator wave-functions for $\mathscr{R}_{2 s}(r)$ and $\mathscr{R}_{1_{p}}(r)$, which apart from normalization are

$$
\left\{\begin{array}{l}
\mathscr{R}_{2 s}=\left[\frac{3}{2}-(r / b)^{2}\right] \exp \left[-\frac{1}{2}(r / b)^{2}\right], \\
\mathscr{R}_{1 p}=(r / b) \exp \left[-\frac{1}{2}(r / b)^{2}\right] .
\end{array}\right.
$$

From the wave functions (12), we obtain the radial integral of eq. (11)

$$
I_{1}=\frac{1}{12} v b\left[(\nu b)^{2}-4\right] \exp \left[-\frac{1}{4}(\nu b)^{2}\right] .
$$


The scale parameter $b$ is fixed by the r.m.s. radius $R_{m}$ of the charge distribution determined from Stanford scattering experiments $\left.{ }^{8}\right)$. For ${ }^{16} \mathrm{O}$ we obtain in the harmonic oscillation model:

$$
b=\frac{2}{3} R_{m}=1.80 \text { fermi } .
$$

The capture rate $1 / \tau$ is given by

$$
\frac{1}{\tau}=\frac{\nu^{2}}{2 \pi}\left|\varphi_{\mu}\right|_{\text {ar }}^{2} \sum_{0_{\text {and }} 1^{-}}\left|M_{\mathrm{ab}}\right|^{2} .
$$

We find from eqs. (10), (13), (14) and (15) that the calculated combined capture rate into the $0^{-}$and $1^{-}$states is approximately $7400 / s$. From this number and from the experimental total capture rate $\left(^{9}\right)$ in ${ }^{16} \mathrm{O}$, we thus expect that approximately $7 \%$ of all the oxygen captures lead to the $0^{-}$and $1^{-}$ states of ${ }^{16} \mathrm{~N}$.

From the summed contributions to the $0^{-}$and $1^{-}$states, eq. (10), we easily obtain the contribution to each state separately.

a) The $0^{+} \rightarrow 0^{-}$transition can have no contribution from the vector part. This is because the initial and final states have opposite parity: thus $\Delta l \neq 0$. Therefore, the total angular momentum change for the vector part $\Delta J=$ $=|\Delta \boldsymbol{l}+\Delta \boldsymbol{s}|=\Delta l \neq 0$, which is incompatible with a $0 \rightarrow 0$ transition. The vector part goes thus entirely into the $1^{-}$state.

b) Take $\hat{v}$ as the $z$-axis; then $\Delta m_{l}=0$ for the orbital operator. The spin-flip operators $\sigma_{x} \boldsymbol{e}_{x}+\sigma_{y} \boldsymbol{e}_{y}=-\sqrt{2}\left(\sigma_{+} \boldsymbol{e}_{-}+\sigma_{-} \boldsymbol{e}_{+}\right)$. of the axial vector term have $\Delta m_{s}= \pm 1$; thus $\Delta M= \pm 1$. They can, therefore, not contribute to a $0 \rightarrow 0$ transition and contribute entirely to the $1^{-}$state.

c) Similarly the no spin-flip operator $(\sigma \cdot \hat{v})=\sigma_{z}\left(=\sigma_{z n}\right)$ cannot contribute to $0^{+} \rightarrow\left(1^{-}, M= \pm 1\right)$, since $\Delta m_{l}=0$ and $\Delta m_{s}=0$ imply $\Delta M=0$. Nor does this term contribute to $\left(1^{-}, M=0\right)$, for, writing the wave functions of eq. (7) as

$$
\begin{aligned}
|I, M=0\rangle & =\left\{\begin{array}{l}
\left|0^{-}\right\rangle \\
\left|1^{-}, M=0\right\rangle
\end{array}\right\}= \\
& =\frac{1}{\sqrt{2}}\left\{\left|1 p_{\frac{1}{2}}^{-1},-\frac{1}{2}\right\rangle_{\mathrm{p}}\left|2 s_{\frac{1}{2}},+\frac{1}{2}\right\rangle_{\mathrm{n}} \mp\left|1 p_{\frac{1}{2}}^{-1},+\frac{1}{2}\right\rangle_{\mathrm{p}}\left|2 s_{\frac{1}{2}},-\frac{1}{2}\right\rangle_{\mathrm{n}}\right\},
\end{aligned}
$$

it is immediately seen that $\sigma_{z n}\left|1^{-}, 0\right\rangle=\left|0^{-}\right\rangle$. $(1959)$.

(8) U. MeYer-Berkhout, K. W. Ford and A. E. S. Green: Ann. of Phys., 8, 119

(9) M. ECKhause: Thesis, Carnegie Institute of Technology, NYO 9286 (1962); J. Barlow, J. C. Sens, P. J. Duke and M. A. R. Kemp: Phys. Lett., 9, 84 (1964). 
Thus the matrix element

$$
\left\langle 0^{+}\left|\exp [-i \nu z] \sigma_{z n}\right| 1^{-}, 0\right\rangle=\left\langle 0^{+}|\exp [-i \nu z]| 0^{-}\right\rangle=0
$$

since it is exactly the matrix element-which occurs in the vector $0^{+} \rightarrow 0^{-}$ transition. The $1^{-}$state, therefore, does not contribute to the no spin-flip transition, which goes entirely into the $0^{-}$state.

From eq. (10) we therefore have without further calculation:

$$
\left\{\begin{array}{l}
\left|M_{0^{+} \rightarrow 0^{-}}\right|^{2}=2\left|I_{1}\right|^{2} \cdot\left(G_{P}-G_{A}\right)^{2} \\
\left|M_{0^{+} \rightarrow 1^{-}}\right|^{2}=2\left|I_{1}\right|^{2} \cdot\left(G_{V}^{2}+2 G_{A}^{2}\right)
\end{array}\right.
$$

and their ratio is thus

$$
\frac{\left|M_{0^{+} \rightarrow 0^{-}}\right|^{2}}{\left|M_{0^{+} \rightarrow 1^{-}}\right|^{2}}=\frac{\left(G_{P}-G_{A}\right)^{2}}{G_{V}^{2}+2 G_{A}^{2}}=\frac{\lambda^{2}[1+x(1-\varrho)]^{2}}{(1+x)^{2}+2(\lambda+\mu x)^{2}}=0.29(1.05-0.05 \varrho)^{2},
$$

in agreement with Ref. $\left({ }^{4}\right)\left(^{*}\right)$. Within the approximations made, the ratio (18) is thus independent of nuclear matrix elements.

The remarkable feature of eq. (18) is the great sensitivity of this ratio to the pseudoscalar coupling constant. Indeed, if the quantity $g_{P} / g_{A}=\varrho$ takes the values $-8,0$ and 8 , the corresponding ratios are $0.61,0.32$ and 0.12 , i.e., a variation by a factor 5 , to be compared with a variation of the order of $60 \%$ if vector or axial vector spin-flip terms had contributed to the $0^{-}$term. It is very interesting to determine the exact conditions for such a maximizing effect in a systematic manner so as to be able to search for other similar cases. We thus consider the capture from a nucleus of spin $I_{\mathrm{a}}$ into a state $I_{\mathrm{b}}$ with relative parity $\pi$. A contribution from vector terms requires $\left|I_{\mathrm{a}}-I_{\mathrm{b}}\right| \leqslant \Delta l \leqslant$ $\leqslant I_{\mathrm{a}}+I_{\mathrm{b}}$ and $\pi=(-)^{\Delta \iota}$. For this to be forbidden in all $\Delta l\left(^{* *}\right)$, we must have a unique $\Delta l$ with $\pi=(-)^{\Delta l+1}$. Therefore, one of $I_{\mathrm{a}}$ or $I_{\mathrm{b}}$ must be 0 ; for the sake of argument say $I_{\mathrm{a}}$. The axial vector spin-flip terms have $\Delta m_{s}= \pm 1$, $\Delta m_{l}=0$, i.e., $\Delta M= \pm 1$. Applied to a zero-spin state they give contributions for $I_{\mathrm{b}} \geqslant 1$. To forbid these contributions we conclude that $I_{\mathrm{b}}=0$ is the only possibility; in addition, the relative parity must be $\pi=(-)^{I_{b}+1}=-1$. The maximum effects of the pseudoscalar term thus occur only in $0^{+} \rightarrow 0^{--}$(or $0^{-} \rightarrow 0^{+}$) captures. Such transitions can be found in a number of cases through

(*) This reference contains some misprints which have been corrected.

(*) We require complete forbiddenness, since higher multipole-order matrix elements in muon capture do not rapidly decrease with increasing $\Delta l$ like in $\beta$-decay. 
the periodic system but the theoretical interpretation will, in general, be hampered by a lack of knowledge of the radial integrals.

\section{4. - Effect of velocity terms and admixtures.}

41. Velocity terms. - So far the nucleon velocity terms in the effective Hamiltonian have been neglected. The leading-order matrix element is firstforbidden in $\beta$-decay terminology (parity change); while forbiddenness does not reduce muon capture matrix elements strongly, it might be suspected that relativistic corrections are more important than usual. This is borne out by our calculation below, particularly for the $0^{-}$state $\left(^{*}\right)$. As a consequence the argument of Shapiro-Blokhintsev, that the $\left(0^{+} \rightarrow 0^{-}\right) /\left(0^{+} \rightarrow 1^{-}\right)$ratio is modelindependent, will fail and it has to be replaced by predictions of absolute rates. By arguments similar to those of Sect. 3, we obtain, from eq. (2), the general expression for the squared matrix in transitions to the $0^{-}$and $1^{-}$states as well as to the $2^{-}$ground state:

$$
\begin{aligned}
& M_{0^{+} \rightarrow 0^{-}}^{2}=\left(G_{P}-G_{A}\right)^{2}\left|\int \sigma_{0}\right|^{2}+2\left(G_{P}-G_{A}\right) g_{A}\left(\int \sigma_{0}\right)^{*}\left(\int \frac{\sigma \cdot p}{M}\right)+g_{A}^{2}\left|\int \frac{\sigma \cdot p}{M}\right|^{2} \\
& M_{0^{+} \rightarrow 1^{-}}^{2}=G_{V}^{2}\left|\int 1\right|^{2}+G_{A}^{2}\left\{\left|\int \sigma_{+}\right|^{2}+\left|\int \sigma_{-}\right|^{2}\right\}-2 g_{V} G_{V} \operatorname{Re}\left(\int 1\right)^{*}\left(\int \frac{p_{0}}{M}\right)+ \\
& +2 g_{V} G_{A} \operatorname{Re}\left\{\left(\int \sigma_{+}\right)\left(\int \frac{p_{+}}{M}\right)^{*}-\left(\int \sigma_{-}\right)\left(\int \frac{p_{-}}{M}\right)^{*}\right\}+g_{V}^{2}\left|\int \frac{\boldsymbol{p}}{M}\right|^{2} \\
& M_{0^{+} \rightarrow 2^{-}}^{2}=G_{A}^{2}\left\{\left|\int \sigma_{+}\right|^{2}+\left|\int \sigma_{-}\right|^{2}\right\}+\left(G_{P}-G_{A}\right)^{2}\left|\int \sigma_{0}\right|^{2}+ \\
& +2 g_{V} G_{\Delta} \operatorname{Re}\left\{\left(\int \sigma_{+}\right)\left(\int \frac{p_{+}}{M}\right)^{*}-\left(\int \sigma_{-}\right)\left(\int \frac{p_{-}}{M}\right)^{*}\right\}+g_{A}^{2}\left|\int \frac{\boldsymbol{\sigma} \cdot \boldsymbol{p}}{M}\right|^{2}+g_{\nabla}^{2}\left|\int \frac{\boldsymbol{p}}{\boldsymbol{M}}\right|^{2},
\end{aligned}
$$

with

$$
\sigma_{ \pm}=\mp \frac{1}{\sqrt{2}}\left(\sigma_{x} \pm i \sigma_{y}\right)
$$

$\sigma_{0}=\sigma_{z}$ and correspondingly for $p_{+}, p_{-}$and $p_{0}$. The $z$-axis is taken along $\hat{\nu}$.

(*) A similar calculation has been done in ref. ${ }^{(0)}$. Our rate to the $1^{-}$state is $30 \%$ lower than found in this reference.

(10) I. Duck: Nucl. Phys., 35, 27 (1962). 


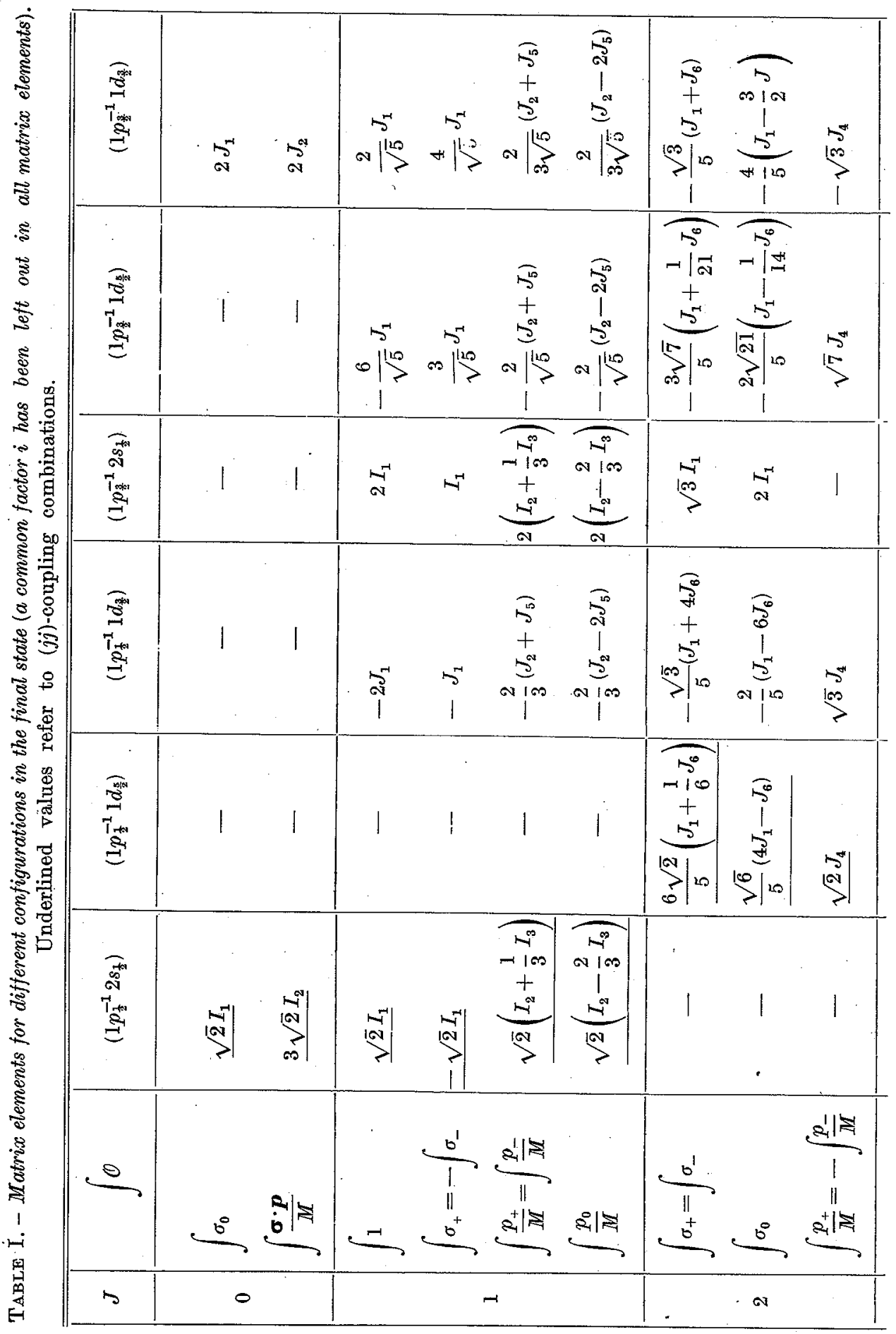


The integrals $f \mathcal{O}$ of eq. (19) are given in Table I, expressed in terms of the radial integrals $I_{i}$ and $J_{i}$. The explicit forms of these integrals are found in Table II for harmonic-oscillator wave-functions. The underlined expressions in Table I refer to transitions in the case of no admixtures to (jj)-coupling; the others correspond to those arising from admixture of other states within the same major oscillator shell of the ${ }^{16} \mathrm{~N}$ wave-functions. The admixture terms will be discussed below.

TABLE II. - The analytical expression for the radial integrals occurring in the matrix elements (Table $\mathrm{I}$ ). In the final column we have omitted a factor $\exp \left[-\frac{1}{4} p^{2}\right]$. Notation: $q \equiv 1 / M b, p \equiv \nu b$, the spherical Bessel functions $j_{l}(\nu r) \equiv j_{l}$.

\begin{tabular}{|c|c|c|}
\hline$I_{1}$ & $\int R_{2 s} j_{1} R_{1 p} r^{2} \mathrm{~d} r$ & $-\frac{1}{3} p\left(1-\frac{1}{4} p^{2}\right)$ \\
$I_{2}$ & $\frac{1}{3 M} \int R_{2 s} j_{0}\left(\frac{2}{r}+\frac{\partial}{\partial r}\right)\left(R_{1 p}\right) r^{2} \mathrm{~d} r$ & $\frac{1}{3} q\left(1-\frac{1}{12} p^{2}+\frac{1}{24} p^{4}\right)$ \\
$I_{3}$ & $\frac{1}{M} \int R_{2 s} j_{2} r \frac{\partial}{\partial r}\left(\frac{1}{r} R_{1 p}\right) r^{2} \mathrm{~d} r$ & $\frac{1}{3} q p^{2}\left(1-\frac{1}{8} p^{2}\right)$ \\
$J_{1}$ & $\int R_{1 a} j_{1} R_{1 p} r^{2} \mathrm{~d} r$ & $\frac{1}{3}\left(\frac{5}{2}\right)^{\frac{1}{2}} p\left(1-\frac{1}{10} p^{2}\right)$ \\
$J_{2}$ & $\frac{1}{M} \int R_{1 d} j_{0} r \frac{\partial}{\partial r}\left(\frac{1}{r} R_{1 p}\right) r^{2} \mathrm{~d} r$ & $-\left(\frac{5}{2}\right)^{\frac{1}{2}} q\left(1-\frac{1}{3} p^{2}+\frac{1}{60} p^{4}\right)$ \\
$J_{4}$ & $\frac{1}{M} \int R_{1 d} j_{2} \frac{1}{r} R_{1 p} r^{2} \mathrm{~d} r$ & $\left(\frac{2}{5}\right)^{\frac{1}{2}} \frac{1}{6} q p^{2}$ \\
$J_{5}$ & $\frac{1}{M} \int R_{1 a} j_{2}\left(\frac{1}{2 r}+\frac{\partial}{\partial r}\right)\left(R_{1 p}\right) r^{2} \mathrm{~d} r$ & $-\left(\frac{2}{5}\right)^{\frac{1}{2}} \frac{7}{6} q p^{2}\left(1-\frac{1}{14} p^{2}\right)$ \\
$J_{6}$ & $\int R_{1 a} j_{3} R_{1 p} r^{2} \mathrm{~d} r$ & $\frac{1}{6} \frac{1}{(10)^{\frac{1}{2}}} p^{3}$
\end{tabular}

Numerical values of the transition rates with and without velocity terms. but without admixtures are given in the first two lines of Table III. It is seen that the influence of velocity terms on the $1^{-}$and $2^{-}$states is small $(-17 \%$ and $-2 \%)$, while the effect on the $0^{-}$state is very large $\left(200 \%\right.$ for $\left.g_{p} / g_{A}=8\right)$. The big effect is mainly due to the small value of $\left(G_{P}-G_{A}\right)$ in this case. The main part of the $0^{-}$matrix element comes thus from the $p / M$ term of eq. (2). In this case the major part of $(p / M)^{2}$ terms are correctly accounted for by the term $A_{2}$ in eq. (2) and they amount to $\approx 20 \%$. 
TABLE III. - Dependence of the partial transition rates ${ }^{16} \mathrm{O}\left(0^{+}\right) \rightarrow^{16} \mathrm{~N}\left(0^{-}, \mathrm{I}^{-}\right.$and $\left.2^{-}\right)$for various values of $g_{P} / g_{A}$ on nucleon velocity terms and admixtures to the ${ }^{16} \mathrm{~N}$ wave-functions. Values given in parentheses refer to rates excluding weak magnetism. The experimental rates are obtained from ref. $\left({ }^{11}\right)$ and ref. $\left({ }^{9}\right)$. The coupling constants used are $g_{A} / g_{V}=-1.20 ; g_{V} \simeq g_{V}^{\beta}=1.42$. $\cdot 10^{-49} \mathrm{erg} \mathrm{cm}^{3}$. The neutrino momentum $\nu$ is $95 \mathrm{MeV} / \mathrm{c}$. The effect of finite nuclear size on the muon wave-function is accounted for by taking $\left|\varphi_{\mu}\right|_{\mathrm{av}}^{2} /\left|\varphi_{\mu_{0}}\right|^{2}=0.79$.

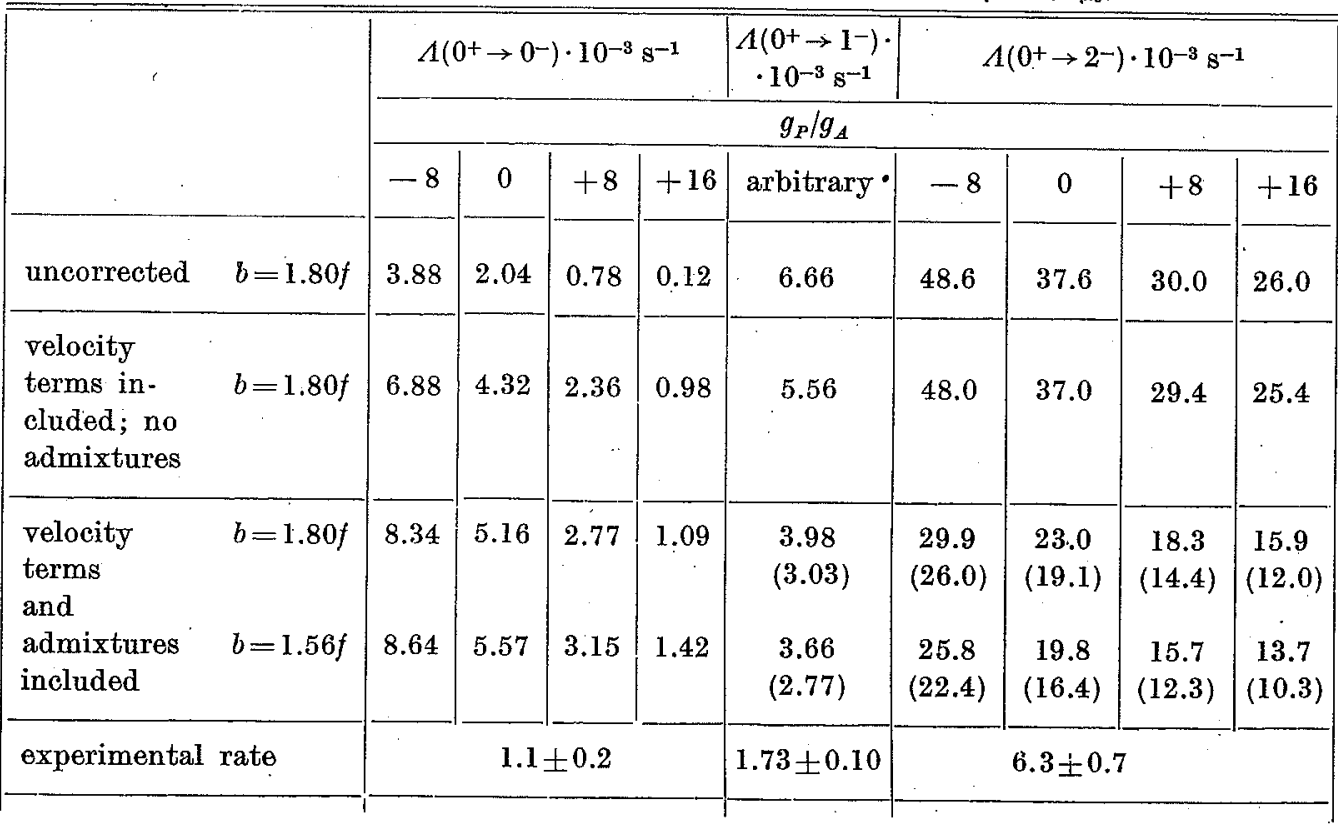

42. Admixtures. - The wave-functions of the $0^{-}$and $1^{-}$states in ${ }^{16} \mathrm{~N}$ have so far. been described by pure (jj)-coupling of $\left|1 p_{\frac{1}{2}}^{-1}\right\rangle_{\mathrm{p}}\left|2 s_{\frac{1}{2}}\right\rangle_{\mathrm{n}}$, while the $2^{-}$state similarly has been expressed by the $\left|1 p_{\frac{1}{2}}^{-1}\right\rangle_{\mathrm{p}}\left|1 d_{\frac{5}{2}}\right\rangle_{\mathrm{n}}$ coupling.

TABLE IV. - Principal wave-function admixtures for low-lying states in ${ }^{16} \mathrm{~N}$ according to ElliotT and Fuowers ( $\left.{ }^{7}\right)$.

\begin{tabular}{|c|c|c|c|c|c|c|}
\hline \hline$I$ & $\left(1 p_{\frac{1}{2}}^{-1} 2 s_{\frac{1}{2}}\right)$ & $\left(\mathrm{l} p_{\frac{1}{2}}^{-1} 1 d_{\frac{5}{2}}\right)$ & $\left(1 p_{\frac{1}{2}}^{-1} 1 d_{\frac{2}{2}}\right)$ & $\left(1 p_{\frac{1}{2}}^{-1} 2 s_{\frac{1}{2}}\right)$ & $\left(1 p_{\frac{1}{2}}^{-1} 1 d_{\frac{5}{2}}\right)$ & $\left(1 p_{\frac{1}{2}}^{-1} 1 d_{\frac{2}{2}}\right)$ \\
\hline $0^{-}$ & 1.00 & - & - & - & - & -0.05 \\
$1^{-}$ & 0.98 & - & 0.01 & -0.16 & -0.08 & -0.02 \\
$2^{-}$ & - & -0.98 & 0.10 & -0.06 & -0.14 & -0.09 \\
$3^{-}$ & - & 0.98 & - & - & -0.18 & 0.06 \\
\hline
\end{tabular}

Other negative pwrity combinations of shell-model wave-functions from the same major oscillator shells must be expected to occur as admixtures. The

(11) R. Cohen, S. Devons and A. D. Kanaris: Phys. Rev. Lett., 11, 134 (1963); corrected in Nucl. Phys., to appear. 
proton hole can be taken as $\left|1 p_{\frac{3}{3}}^{-1}\right\rangle_{\mathbf{p}}$ and $\left|1 p_{\frac{1}{2}}^{-1}\right\rangle_{\mathrm{p}}$; the neutron in the higher oscillator-shell can be $\left|2 s_{\frac{1}{2}}\right\rangle_{\mathrm{n}},\left|1 d_{\frac{5}{2}}\right\rangle_{\mathrm{n}}$ and $\left|1 d_{\frac{3}{2}}\right\rangle_{\mathrm{n}}$.

The admixture amplitudes of these configurations have been calculated by ELLTOTT and Fuowers $\left({ }^{7}\right)$ by taking into account the interactions between the nucleons (see Table IV). The smallness of the admixture amplitudes to pure $(j j)$-coupling is apparent.

We have calculated the matrix elements corresponding to these admixture configurations (including the contribution from velocity terms). They are listed in Table I expressed in the radial integrals $I_{i}, J_{i}$ of Table II. By multiplying these normalized matrix elements with their corresponding admixture amplitude (see for example Table IV) and adding, the correct integrals $\int \mathcal{O}$ of Eq. (19) are obtained.

Numerical values of the transition rates for Elliott and Flowers' wave functions are given in Table III for two different radii. The rates depend nonnegligibly on admixtures with a change of the $0^{-}, 1^{-}$and $2^{-}$rates by $22 \%, 28 \%$ and $36 \%$, respectively, as compared to no admixtures with velocity terms included.

\section{5. - Conclusions.}

In the previous Sections we have considered the influence of nucleon velocity terms and wave-function admixture on the partial transitions to the $0^{-}$, $1^{-}$and $2^{-}$states of ${ }^{16} \mathrm{~N}$.

1) We find that the velocity terms dominate the "leading " contribution to the $0^{+} \rightarrow 0^{-}$transition and give effects of the order of $200 \%$ for relevant values of $g_{P}$. The reason for this large influence is the smallness of the apparent coupling constant $\left(G_{P}-G_{A}\right)$ in the "leading " term for large positive values of $g_{p}$. The effect of velocity terms on the other two transitions is small.

2) Contrary to the assumptions of BLOKHINTSEV and SHAPIRo, admixtures to the ${ }^{16} \mathrm{~N}$ wave-functions cannot be neglected. Our calculation of admixture effects should not be considered as a precise evaluation but as a general indication of the sign and magnitude of these corrections.

3) In the original calculation of BLOKHTNTSEV and SHAPIRO neglecting corrections, the ratio $\left(0^{+} \rightarrow 0^{-}\right) /\left(0^{+} \rightarrow 1^{-}\right)$became independent of detailed nuclear structure since both rates were described by the same radial matrix element. The large influence of velocity terms with "different radial matrix elements and of admixtures prevents this cancellation and introduces model dependence. Hence results obtained from this ratio should be considered to be rather uncertain. A comparison of the calculated ratio with the results of 
present experiments $\left({ }^{11,12}\right)$ in Fig. 2 points to a positive $g_{P} / g_{A}$ consistent with a value of the order of 8 . This should be compared to the results of radiative capture $\left({ }^{2,3}\right)$, neutron asymmetry $\left(^{1}\right)$ and capture in hydrogen $\left({ }^{12}\right)$ which indicate a value of $g_{p}$ larger than the predicted one.

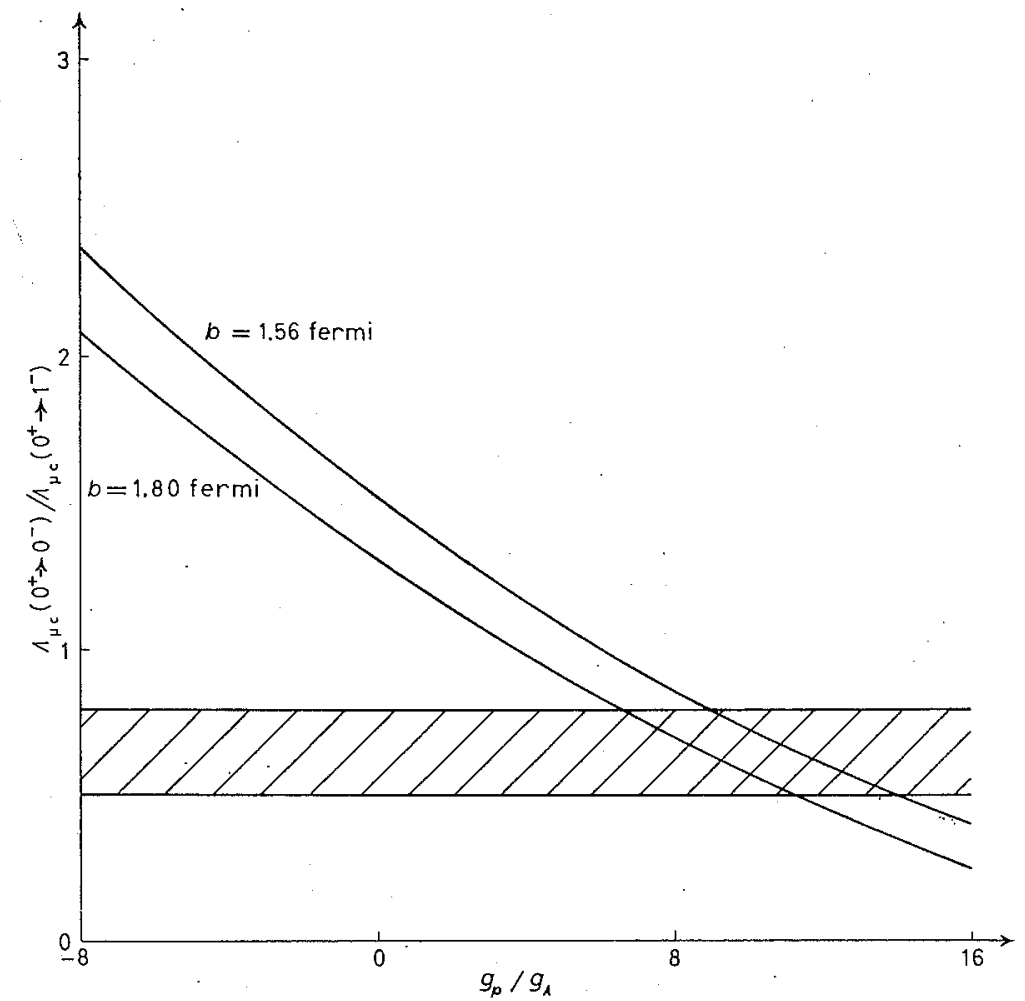

Fig. 2. - The ratio of the transition rates $0^{+} \rightarrow 0^{-}$and $0^{+} \rightarrow 1^{-} v s . g_{P} / g_{A}$ for two values of the radial parameter $b$. The shaded area indices the experimental ratio \pm one standard deviation (see ref. $\left.{ }^{(11}\right)$ ).

4) While the uncorrected $0^{+} \rightarrow 0^{-}$rate changes by a factor 5 for $g_{P} / g_{A}$ going from -8 to +8 the corrected rate has its variation reduced to about a factor 3 .

5) The absolute predicted rates into the $0^{-}, 1^{-}$and $2^{-}$states are all about a factor 2 larger than the results of two recent experiments $\left({ }^{11,12}\right)$ (see Table III). They do, however, somewhat favour a relatively large positive value $g_{P}$.

6) In the case of the $2^{--}$transition the nuclear matrix element can be experimentally determined from the corresponding $2^{-} \rightarrow 0^{+}$unique first-forbid-

(14) A. Astbury, L. Autrbach, D. Cutis, R. Esteriting, D. Jenkins, N. LipMAN, R. SHAFER: UCRL.11115 (19E4). 
den $\beta$-decay, but for a form factor correction of about $20 \%$ and for velocity terms of a few percent. Detailed assumptions about nuclear radius and wavefunction admixtures have then only to be made in terms which are already small. The calculated $\beta$-decay rate for $b=1.80$ and $E_{\beta \max }=10.40 \mathrm{MeV}$ is

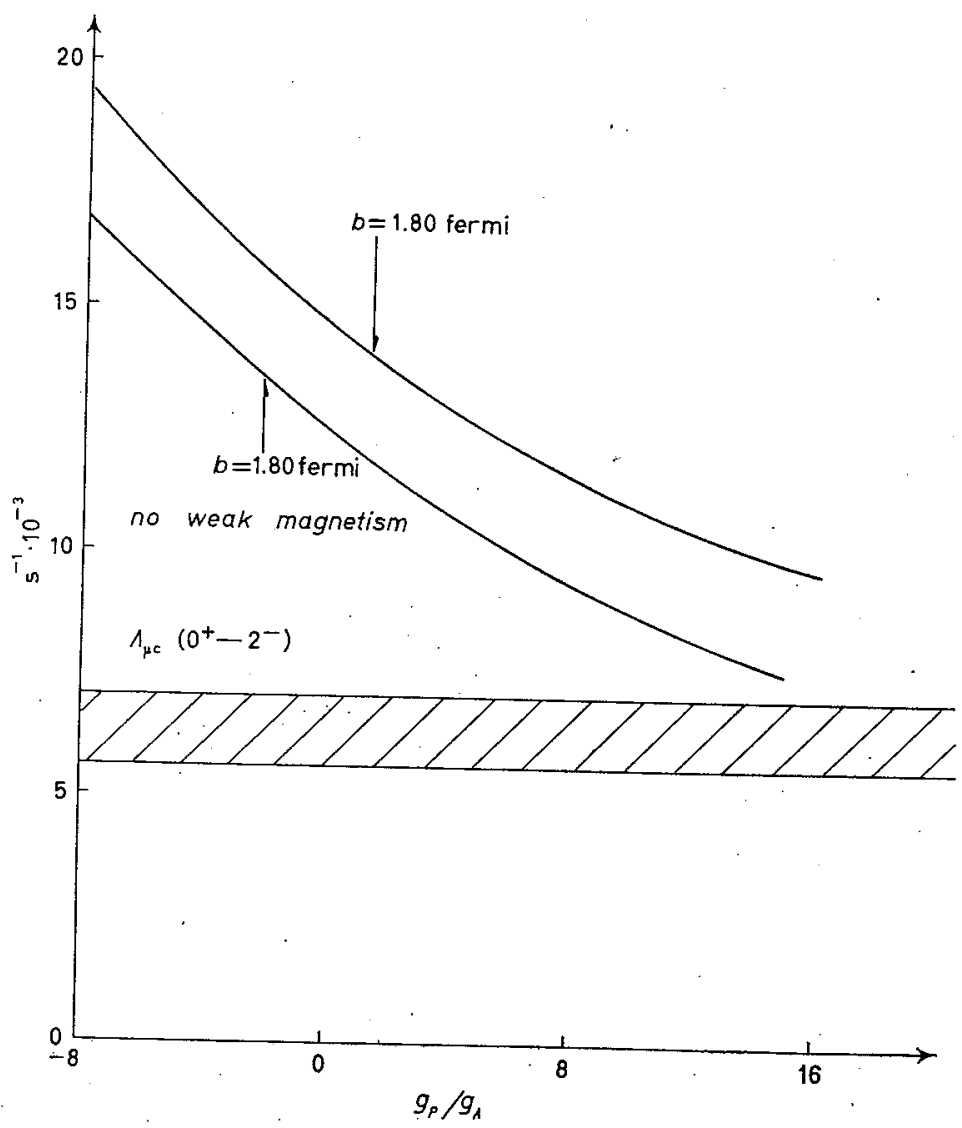

Fig. 2. - The partial transition rate $0^{+} \rightarrow 2^{-}$as a function of $\varrho=g_{P} / g_{A}$. In the lower curve the weak magnetism term is omitted. For both curves the radial parameter $b=1.80$ fermi. Both curves are corrected by the ratio of the experimental to the theoretical value of the $2^{-} \rightarrow 0^{+} \beta$-decay rate. The shaded area indicates the experimental value \pm one standard deviation [see ref. (11)].

$3.91 \cdot 10^{-2} \mathrm{~s}^{-1}$ to be compared with an experimental rate $(2.45 \pm 0.20) \cdot 10^{-2} \mathrm{~s}^{-1}$. The calculated $2^{-}$capture rate in Table III should hence be multiplied by the ratio $\Gamma_{\beta}(\exp ) / \Gamma_{\beta}$ (theor) $=2.45 / 3.91=0.61$. The capture rates so obtained are basically trustworthy to $(5 \div 10) \%$. The major sources of uncertainty in the rate obtained in this way are: $a$ ) the $\beta$ decay experimental branching ratio $[ \pm 8 \%] ; b)$ the experimental $\beta$ end-point energy $[ \pm 3 \%]$; and $c$ ) the average 
muon wave-function (a few \%). With this experimentally determined nuclear matrix element the $2^{-}$rate becomes an excellent detector for the combination $\approx\left[2 G_{A}+\left(G_{P}-G_{A}\right)^{2}\right]$ of the coupling constants. The experimental rate ( $\left.{ }^{13}\right)$ suggests a large value of $g_{P}$ (larger than $\approx 13 g_{A}$ ). Due to the conflicting experimental evidence, we do not feel justified in drawing any further conclusion from the experimental material at present.

The authors wish to express their gratitude to Prof. H. A. ToLHOEK for helpful discussions.

\section{RIASSUNTO (*)}

La rivelazione dei rapporti parziali di cattura muonica nel ${ }^{16} \mathrm{O}$ costituisce un sensibile rivelatore per la costante di accoppiamento pseudoscalare indotto. Qui si studiano gli effetti sull'hamiltoniana debole dei termini contenenti la velocità del nucleone e delle funzioni d'onde nucleari realistiche. La loro inclusione è essenziale per una corretta trattazione del problema. In aggiunta al livello di rivelazione suggerito precedentemente, si trova che la cattura nello stato fondamentale del ${ }^{16} \mathrm{~N}$ permette una eliminazione quasi completa della fisica nucleare in confronto al rapporto di decadimento $\beta$.

(") Traduzione a cura della Redazione. 